\title{
Two- and Four-Way Coupled Euler-Lagrangian Large-Eddy Simulation of Turbulent Particle-Laden Channel Flow
}

\author{
Bert Vreman • Bernard J. Geurts • N. G. Deen • \\ J. A. M. Kuipers • J. G. M. Kuerten
}

Received: 8 October 2007 / Accepted: 22 July 2008 / Published online: 26 August 2008

(C) The Author(s) 2008

\begin{abstract}
Large-eddy simulations (LES) of a vertical turbulent channel flow laden with a very large number of solid particles are performed. The motivation for this research is to get insight into fundamental aspects of co-current turbulent gas-particle flows, as encountered in riser reactors. The particle volume fraction equals about $1.3 \%$, which is relatively high in the context of modern LES of two-phase flows. The channel flow simulations are based on large-eddy approximations of the compressible Navier-Stokes equations in a porous medium. The Euler-Lagrangian method is adopted, which means that for each individual particle an equation of motion is solved. The method incorporates four-way coupling, i.e., both the particle-fluid and particle-particle interactions are taken into account. The results are compared to single-phase channel flow in order to investigate the effect of the particles on
\end{abstract}

B. Vreman

Vreman Research, Godfried Bomansstraat 46,

7552 NT Hengelo, The Netherlands

\section{B. J. Geurts $(\bowtie)$}

Multiscale Modeling and Simulation, Department of Applied Mathematics, University of Twente, P.O. Box 217, 7500 AE Enschede, The Netherlands

e-mail: b.j.geurts@utwente.nl

\section{B. J. Geurts}

Anisotropic Turbulence, Fluid Dynamics Laboratory,

Eindhoven University of Technology, P.O. Box 513,

5300 MB Eindhoven, The Netherlands

N. G. Deen · J. A. M. Kuipers

Department of Chemical Engineering, J.M. Burgers Center, Faculty TNW, University of Twente, P.O. Box 217,

7500 AE Enschede, The Netherlands

J. G. M. Kuerten

Department of Mechanical Engineering,

J.M. Burgers Center, Technische Universiteit Eindhoven, P.O. Box 513,

5600 MB Eindhoven, The Netherlands 
turbulent statistics. The present results show that due to particle-fluid interactions the mean fluid profile is flattened and the boundary layer is thinner. Compared to single-phase turbulent flow, the streamwise turbulence intensity of the gas phase is increased, while the normal and spanwise turbulence intensities are reduced. This finding is generally consistent with existing experimental data. The four-way coupled simulations are also compared with two-way coupled simulations, in which the inelastic collisions between particles are neglected. The latter comparison clearly demonstrates that the collisions have a large influence on the main statistics of both phases. In addition, the four-way coupled simulations contain stronger coherent particle structures. It is thus essential to include the particle-particle interactions in numerical simulations of two-phase flow with volume fractions around one percent.

Keywords Turbulence $\cdot$ Particle-laden flow $\cdot$ Large-eddy simulation • Channel flow • Inelastic collisions • Coherent structures · Four-way coupling • Turbulence modulation

\section{Introduction}

Many flows of relevance to large-scale chemical processing involve solid catalyst particles at significant concentrations embedded in a carrying gas-flow. Control over the spatial distribution of these particles, especially its homogeneity, is essential in order to provide a chemical processing that is as complete and uniform as possible, that is consistent with modern environmental requirements and that does not constitute a strong safety hazard. This provides the main context for this study which is directed toward understanding the fundamental aspects of the dynamics of the embedded, interacting particles, and to develop a simulation strategy with which the central up-scaling of laboratory-scale experiments to realistic industrial settings can be supported.

The dynamics of the embedded particle-ensemble is quite complex and interacts nonlinearly with the carrying gas-flow. The particles are dragged along by this carrying gas-flow and exchange momentum with it. Moreover, the solid particles interact among each other, e.g., through inelastic particle-particle collisions. In case only particle-fluid interactions are incorporated the description is referred to as 'twoway coupled' while a 'four-way coupled' formulation arises when also the particleparticle interactions are included. At sufficiently low particle volume fraction $\psi$ two-way coupling is adequate. However, with increasing $\psi$ the collisions will become dynamically significant and the computationally more involved four-way coupling will become required. The main purpose of this paper is to demonstrate in the context of numerical simulation that at a realistic mass load ratio of 19.5 and a particle volume-fraction of $1.3 \%$ the collisions constitute a major dynamic effect that needs to be incorporated in order to retain a physically reliable flow description. This forms a separate confirmation of the commonly accepted classification in which volume-fractions larger than $10^{-3}$ necessitate the inclusion of particle-particle collisions [8]. The present study is the first to establish the relevance of the particleparticle collisions, at moderately high volume fractions, using detailed turbulence 
simulations. We will show that these collisions strongly influence the main statistical fluid properties and amplify the 'self-organization' of the embedded particles in coherent swarms.

The two-phase gas-solid flow is governed by an interplay between the convective gas-flow nonlinearity, the particle-fluid and the particle-particle interactions. These effects may accumulate and significantly change basic turbulence properties such as mean flow and turbulence intensities. A large-scale dynamic flow-structuring may arise affecting the flow-statistics compared to the case with no or only weak interactions. These flow-alterations constitute the so-called modulation of turbulence (see [20]) which, e.g., seriously complicates the prediction of the up-scaling of flowphenomena from laboratory-scale experiments to industrial-scale settings. We will consider large-eddy simulations of a vertical gas-solid channel flow to support research in this problem-area and to help understand the fundamental modulation of the turbulent flow properties.

The design of large-scale chemical processes is hampered by the lack of precise (design) tools which capture the dynamics in systems of realistic proportions. Since full-scale experimental research is costly and often not precise or not feasible, the development of accurate simulation tools is very important. A specific example is the cracking of oil which is facilitated by adding large numbers of catalyst particles to a carrying gas-flow. Basic to catalytic cracking is an understanding of the granular dynamics of large swarms of grains of sand. Specifically, it is important to investigate whether particle-particle collisions are dynamically important and lead to large clusters, thus contributing to spatial non-uniformities that may jeopardize safety and product-consistency and increase pollution.

Turbulent gas-solid flows have been studied experimentally (e.g., [30, 43, 46, 59]) and with simulations. Simulations can be performed using a two-fluid model in which the solid phase is modeled as a fluid using continuous variables (e.g., $[19,38,46]$ ). This approach is quite well established and may be used to investigate statistical properties of multi-phase flows [12]. A promising, more recent, direction to solve two-phase flows is to enforce the no-slip condition on the boundary of each particle using front tracking methods (see e.g., $[10,58]$ ). No additional modeling assumptions are required, but the amount of particles that can be calculated is currently around 1,000. In this paper we consider a third approach, the discrete particle method in which the Navier-Stokes equations which govern the fluid in a Eulerian framework are combined with a Lagrangian tracking of the motion of each individual particle. The forces between the fluid and each particle are modeled with a drag law and all collisions between particles are treated with a deterministic approach [24, 34]. The modeling is more refined than in two-fluid models, while it presently allows 100-1,000 times more particles than the direct front tracking methods.

Bagchi and Balachandar [3] investigated the validity of the standard drag law for particles with a diameter $1.5 \eta<d<10 \eta$, where $\eta$ is the Kolmogorov length-scale. They found that the time-averaged drag is accurately predicted and insensitive to whether the fluid velocity is measured at the particle center, or obtained by averaging over a fluid volume of the order of the particle size. Instantaneous drag is reasonably well predicted for moderate particle sizes, e.g., $d<4 \eta$. The diameter of the particles in the present study equals $2 \eta$ and is within this region. Hence, we will assume the drag law to be adequately representative of the dominant particle-motion physics. 
The discrete particle technique will be combined with large-eddy simulation (LES) of the fluid flow. Large-eddy simulation solves the large flow scales like direct numerical simulation (DNS), but models the effect of the small scales with a subgridmodel (see the reviews by Pope [49], Sagaut [53] and Geurts [17]). It is considerably more efficient than direct numerical simulation (DNS), which resolves all turbulent scales in the flow. These techniques are able to give proper detailed descriptions of the turbulence in a channel flow.

LES/DNS of channel flows supplemented with a discrete particle model have been reported a number of times (e.g., [1, 2, 37, 56, 69, 70]). However, the total solid volume fraction in these studies remains rather small $(0.01 \%)$ and most of these works employ one- or two-way coupling. An exception is Yamamoto et al. [69] who started to investigate the influence of particle-particle interactions in LES of channel flow with particle volume fractions up to $0.014 \%$. They found that even in such dilute regimes the effects of collisions are significant. Discrete particle models have also been used for detailed studies of two-phase isotropic turbulence. These studies have been performed with volume fractions up to $0.1 \%$ and were all restricted to two-way coupling $[8,11,55]$. For pipe flow larger solid volume fractions have been achieved recently [64].

The purpose of this paper is to present LES of a channel flow in which the particle volume concentration is an order of magnitude higher than existing EulerLagrangian studies in literature and closer to industrial applications. The discrete particle module developed by Hoomans et al. [24] will be used, in which the spherical particles have a finite size and all (inelastic) collisions are taken into account. A subgrid closure needs to be adopted for the LES-equations of the gas phase for which we will mainly adopt the recently developed model by Vreman [63].

In order to study both the effect of the particle collisions and the effects of the particle-fluid interactions we will compare the following three simulations: (1) a turbulent channel flow without particles, (2) a turbulent channel flow with particles, but without collisions (two-way coupled case) and (3) a turbulent channel flow with colliding particles (four-way coupled case). The differences between cases 1 and 2 quantify the effects of the particle-fluid interactions and the differences between cases 2 and 3 quantify the effects of the particle collisions.

The organization of this paper is as follows. In Section 2 we extensively present the simulation method. Results of a large number of channel flow simulations are presented in Section 3, focusing on turbulence modulation, the differences between two- and four-way coupling and coherent particle structures. Finally, concluding remarks are collected in Section 4.

\section{Mathematical Formulation}

In this section we specify the mathematical formulation of the simulation model for the turbulent gas-solids flow. In Section 2.1 the equations governing the gas-phase are described. The treatment of the solids-phase is specified in Section 2.2. The subgrid modeling for the turbulent stresses that arise in the large-eddy simulation is introduced in Section 2.3 and, finally, the numerical method is discussed in Section 2.4. 


\subsection{The gas phase}

The computational model distinguishes a gas phase and a solids phase. The embedded solid particles are considered to be small compared to convective turbulent length-scales. This allows to effectively approximate the equations for the gas phase in terms of flow through a (time- and position-dependent) porous medium $[24,31,50,72,74]$. The local, instantaneous particle concentration determines the volume-fraction that is accessible to the gas phase. However, although the present particle concentration is considerably higher than in previous Euler-Lagrangian studies of gas-solid channel flow, it is still sufficiently small to omit the porous medium effects in the gas equation. Thus for the gas equation we simply extend the standard Navier-Stokes equations that govern a compressible flow with appropriate forcing terms:

$$
\begin{aligned}
\partial_{t} \rho+\partial_{j}\left(\rho u_{j}\right) & =0, \\
\partial_{t}\left(\rho u_{i}\right)+\partial_{j}\left(\rho u_{i} u_{j}\right) & =-\partial_{i} p+\partial_{j} \sigma_{i j}+\rho a_{\mathrm{ext}} \delta_{i 3}+f_{i}, \\
\partial_{t} e+\partial_{j}\left((e+p) u_{j}\right) & =\partial_{j}\left(\sigma_{i j} u_{i}\right)+\rho a_{\mathrm{ext}} u_{3}+f_{i} u_{i}-\partial_{j} q_{j} .
\end{aligned}
$$

where the symbols $\partial_{t}$ and $\partial_{j}$ denote the partial differential operators $\partial / \partial t$ and $\partial / \partial x_{j}$ respectively. Furthermore, $\rho$ is the density, $\mathbf{u}$ the velocity, $p$ the pressure and $e=p /(\gamma-1)+\frac{1}{2} \rho u_{k} u_{k}$ the total energy per volume unit. The constant $\gamma$ denotes the ratio of specific heats $C_{P} / C_{V}=1.4$. The coordinate $x_{3}$ denotes the streamwise direction of the channel flow, $x_{2}$ is the normal and $x_{1}$ is the spanwise direction. Throughout, we will frequently interchange the symbols $x_{1}, x_{2}$ and $x_{3}$ by $x, y$ and $z$ and $u_{1}, u_{2}$ and $u_{3}$ by $u, v$ and $w$ respectively. The domain is rectangular and the channel width, height and depth equal $L_{2}=0.05 \mathrm{~m}, L_{3}=0.30 \mathrm{~m}$ and $L_{1}=0.075 \mathrm{~m}$ respectively. Periodic boundary conditions are assumed for the stream- and spanwise directions. The resolution of the LES, specified in Section 2.4, is such that we do not need a model for the wall shear stress; we thus impose no-slip boundary conditions for the velocities at the wall. Neumann boundary conditions are used for the temperature at the wall.

The viscous stress $\sigma_{i j}$ equals $2 \rho v S_{i j}$ where $v$ is the fluid viscosity and the strain-rate is defined by

$$
S_{i j}(u)=\frac{1}{2} \partial_{i} u_{j}+\frac{1}{2} \partial_{j} u_{i}-\frac{1}{3} \delta_{i j} \partial_{k} u_{k}
$$

The heat-flux $q_{j}$ is defined as $-\kappa \partial_{j} T$ where $T$ is the temperature and $\kappa$ the heatconductivity coefficient. Pressure, density and temperature are related to each other by the equation of state for an ideal gas $\rho R_{\text {gas }} T=M_{\text {gas }} p$, where $R_{\text {gas }}=8.314 \mathrm{~J} /(\mathrm{mol} \mathrm{K})$ is the universal gas constant and $M_{\text {gas }}=0.0288 \mathrm{~kg} / \mathrm{mol}$ is the molar mass of the gas.

The symbol $a_{\text {ext }}$ represents the acceleration caused by external forces on the gas phase and $f_{i}$ denotes the contributions due to the force of the particles on the flow. These are induced by an effective relative motion of the particles with respect to the gas which gives rise to drag forces on the fluid (see next subsection). In principle the heat transfer between particles and fluid should also be represented in the energy 
equation, but this effect is neglected, because temperature differences play a minor role in the applications we presently have in mind.

We are interested in a section of a riser flow with a vertical centerline velocity $U_{c}$ of about $4 \mathrm{~m} / \mathrm{s}$. The parameters of the fluid in the riser are close to those for air. The initial fluid density is uniform and equals $\rho_{g}=1.0 \mathrm{~kg} / \mathrm{m}^{3}$. The viscosity equals $v=$ $3.47 \cdot 10^{-5} \mathrm{~m}^{2} / \mathrm{s}$ and the heat-conductivity is obtained from the assumption that the Prandtl number equals one. The value of the viscosity is chosen such that $\operatorname{Re}_{\tau}=180$ for channel flow without particles and $U_{c}=4.5 \mathrm{~m} / \mathrm{s}$. The Kolmogorov length-scale in channel flow equals about $\eta^{+} \approx 1.5$ in wall-units [49], which implies $\eta \approx 0.2 \mathrm{~mm}$.

With a typical value of the initial pressure (around $10^{5} \mathrm{~N} / \mathrm{m}^{2}$ ) the flow has a very low Mach number around 0.01. Instead of using an incompressible solver, we use the available compressible LES solver [66] with an adapted initial pressure level such that the Mach number based on $U_{c}$ approximately equals 0.2. At this Mach number the turbulent channel flow can still be regarded as approximately incompressible, which means that a further reduction of the Mach number does not significantly change the turbulent statistics, including pressure fluctuations. The flow is driven by an external acceleration $a_{\text {ext }}$, which represents the combined effect of an external pressure gradient and gravity on the gas phase. This acceleration is a function of time only and its level is such that the total fluid mass flow is constant. In all cases the average mean gas velocity is identical, $U_{m}=3.92 \mathrm{~m} / \mathrm{s}$.

\subsection{The solids phase}

The number of solid particles in the channel flow equals $N_{p}=419,904$. During the simulations the motion of all these particles was tracked, starting from an initially uniform distribution of particles throughout the flow-domain. The initial velocity of each particle was taken equal to the local initial velocity of the gas-phase. The particle diameter and density are $d_{p}=0.4 \mathrm{~mm}$ and $\rho_{p}=1,500 \mathrm{~kg} / \mathrm{m}^{3}$, respectively. With the parameters above the average volume fraction of the particles equals 0.013 . The Stokes response-time, defined as

$$
\tau_{p}=\frac{\rho_{p} d_{p}^{2}}{18 \mu}
$$

equals $0.4 s$. The Stokes number equals 10, based on the Kolmogorov time derived from the average dissipation of the unladen flow. In this paper only a single, rather high, value of the Stokes response-time will be adopted in order to emphasize the dynamic effects of the embedded particles. This provides a characteristic, demanding case of turbulent gas-solid flow which is used to assess the feasibility and accuracy of Euler-Lagrangian LES. A comprehensive parameter-study into the physics of this two-phase flow in which also smaller particles, at lower Stokes response-time and volume fraction, are incorporated, is subject of ongoing research. Such a study necessitates an efficient parallel processing of the computational model. We consider volume fractions of $\approx 1.5 \%$. This is considerably higher than reported thus far in literature. However, it is still sufficiently low to use (1)-(3) as an acceptable approximation for the dynamics of this particle-laden flow.

In the following we formulate the standard drag law and the particle collision model, which govern the solids momentum. The mean velocity of the riser is low 
enough to neglect the heat transfer during particle collisions and the heat transfer between particles and fluid.

The motion of every individual particle $i$ in the system is calculated from Newton's second law:

$$
m_{i} \frac{d \mathbf{v}_{i}}{d t}=V_{i} \beta\left(\mathbf{u}-\mathbf{v}_{i}\right)+m_{i} g \mathbf{e}_{z}+\mathbf{f}_{i}^{\mathrm{pp}}+\mathbf{f}_{i}^{\mathrm{pw}},
$$

where $m_{i}$ denotes the mass, $\mathbf{v}_{i}$ the velocity, $V_{i}$ the volume of the $i$-th particle and $\mathbf{e}_{z}$ is the unit vector in the $z$-direction. The gravitational acceleration equals $g=-9.81 \mathrm{~m} / \mathrm{s}^{2}$, which is opposite to the mean flow direction. The forces on the right hand side of the equation represent standard drag, gravity, particle-particle interaction $\left(\mathbf{f}_{i}^{\mathrm{pp}}\right)$ and particle-wall interaction $\left(\mathbf{f}_{i}^{\mathrm{pw}}\right)$, respectively. The general equation of motion for a single particle derived by Maxey and Riley [39] contains additional forces, such as added mass and history terms. However, the comparison with DNS results performed by Bagchi and Balachandar [3] did not show improvements when these forces were included. In the present case, the particle density is much larger than the fluid density. Correspondingly, these additional forces, including buoyancy effects are relatively small and can be neglected [2]. It is unlikely that collisions between solid particles would alter this finding.

The symbol $\beta$ in the drag term is the inter-phase momentum transfer coefficient. The flow is sufficiently dilute to employ the correlation of Wen and $\mathrm{Yu}$ [71] is used:

$$
\frac{\beta d_{p}^{2}}{\mu}=\frac{3}{4} C_{D} \operatorname{Re} ; C_{D}= \begin{cases}24\left(1+0.15 \mathrm{Re}^{0.687}\right) / \mathrm{Re} ; & \mathrm{Re}<10^{3} \\ 0.44 & \mathrm{Re}>10^{3},\end{cases}
$$

$\operatorname{Re}=\rho\left|\mathbf{u}-\mathbf{v}_{\mathrm{p}}\right| \mathrm{d}_{\mathrm{p}} / \mu$ is the particle Reynolds number, which is evaluated at the particle position. As an alternative the drag closure can be obtained from detailed lattice Boltzmann simulations [22].

The two-way coupling between the gas phase and the particles is achieved via the sink term $\mathbf{f}$ in the momentum equations of the gas phase, which is computed from:

$$
\mathbf{f}(\mathbf{r})=\frac{1}{V_{\text {cell }}} \int_{V_{\text {cell }}} \sum_{i=0}^{N_{p}} V_{i} \beta\left(\mathbf{u}-\mathbf{v}_{i}\right) D\left(\mathbf{r}_{i}-\mathbf{r}\right) d V
$$

where $V_{\text {cell }}$ represents the nonuniform volume of the local fluid grid cell which contains the location vector $\mathbf{r}$ at which the drag term needs to be computed. The distribution function $D$ locally distributes the reaction force acting on the gas phase to the Eulerian grid via volume weighing (see [24] for more details). Note that $D$ is an approximation of the Dirac delta function.

The collision model used in this work is based on the hard-sphere model developed by Hoomans et al. [24] and Hoomans [25], who applied this model to twodimensional flow first. However, the model has also been validated and frequently applied to three-dimensional flows (e.g., [7, 23] and references therein). In the collision model it is assumed that the interaction forces are impulsive and therefore all other finite forces are negligible during collision. Consider two colliding spheres $a$ and $b$ with position vectors $\mathbf{x}_{a}$ and $\mathbf{x}_{b}$. The particle velocities prior-to-collision are indicated by the subscript 0 and the relative velocity at the contact point $c$ (i.e., just after the collision) is defined as $v_{a b}=v_{a, c}-v_{b, c}$. The velocities prior to collisions are the velocities at the last time step before the collision (the corresponding time difference is not larger than $10^{-4} s$ ). 
For a binary collision of these spheres the following equations can be derived by applying Newton's second and third laws:

$$
\begin{gathered}
m_{a}\left(\mathbf{v}_{a}-\mathbf{v}_{a, 0}\right)=-m_{b}\left(\mathbf{v}_{b}-\mathbf{v}_{b, 0}\right)=\mathbf{J}, \\
\frac{I_{a}}{R_{a}}\left(\boldsymbol{\omega}_{a}-\boldsymbol{\omega}_{a, 0}\right)=\frac{I_{b}}{R_{b}}\left(\boldsymbol{\omega}_{b}-\boldsymbol{\omega}_{b, 0}\right)=-\mathbf{n} \times \mathbf{J},
\end{gathered}
$$

where $\boldsymbol{\omega}$ denotes the angular velocity, $\mathbf{J}$ is the momentum vector, $R$ is the particle radius and $\mathbf{n}$ is the unit vector directed along $\mathbf{x}_{a}-\mathbf{x}_{b}$. The moment of inertia $I$ is given by:

$$
I=\frac{2}{5} m R^{2}
$$

Equations 9 and 10 can be rearranged to obtain:

$$
\mathbf{v}_{a b}-\mathbf{v}_{a b, 0}=\frac{7 \mathbf{J}-5 \mathbf{n}(\mathbf{J} \cdot \mathbf{n})}{2 m_{a b}},
$$

where $m_{a b}$ is the reduced mass given by:

$$
m_{a b}=\left(\frac{1}{m_{a}}+\frac{1}{m_{b}}\right)^{-1} .
$$

In order to calculate the post-collision velocities, a closure model consisting of three parameters is used to describe the momentum vector $\mathbf{J}$. The first parameter is the coefficient of normal restitution, $(0 \leq e \leq 1)$ :

$$
\mathbf{v}_{a b} \cdot \mathbf{n}=-e\left(\mathbf{v}_{a b, 0} \cdot \mathbf{n}\right) .
$$

The second parameter is the coefficient of dynamic friction $\left(\mu_{f} \geq 0\right)$ :

$$
|\mathbf{n} \times \mathbf{J}|=-\mu_{f}(\mathbf{n} \cdot \mathbf{J}) .
$$

The third parameter is the coefficient of tangential restitution $\left(0 \leq \beta_{0} \leq 1\right)$ :

$$
\mathbf{v}_{a b} \cdot \mathbf{t}=-\beta_{0}\left(\mathbf{v}_{a b, 0} \cdot \mathbf{t}\right)
$$

Combining (12) and (14) yields the following expression for the normal component of the momentum vector:

$$
J_{n}=-(1+e) m_{a b} \mathbf{v}_{a b, 0} \cdot \mathbf{n}
$$

For the tangential component, two types of collisions can be distinguished, i.e. sticking or sliding collisions. If the tangential component of the relative velocity is sufficiently high in comparison to the coefficients of friction and tangential restitution, gross sliding occurs throughout the whole duration of the contact and the collision is of the sliding type. The non-sliding collisions are of the sticking type. When $\beta_{0}$ is equal to zero, the tangential component of the relative velocity becomes zero during a sticking collision. When $\beta_{0}$ is greater than zero in such a collision, reversal of the 
Table 1 Parameters used in the treatment of the discrete particles

\begin{tabular}{lll}
\hline Symbol & Value & Description \\
\hline$d_{p}$ & $410^{-4} \mathrm{~m}$ & Particle diameter \\
$\rho_{p}$ & $1.510^{3} \mathrm{~kg} \mathrm{~m}^{-3}$ & Particle density \\
$N_{p}$ & 419904 & Number of particles \\
$e$ & 0.97 & Normal restitution coefficient \\
$\beta_{0}$ & 0.33 & Tangential restitution coefficient \\
$\mu_{f}$ & 0.1 & Friction coefficient \\
\hline
\end{tabular}

Each particle is spherical, corresponds to a Stokes response-time $\tau_{p}=0.4 \mathrm{~s}$, associated with a viscosity of the gas phase of $\mu=3.4710^{-5} \mathrm{~kg} \mathrm{~m}^{-1} \mathrm{~s}^{-1}$. The solids phase has an average volume fraction $\langle\psi\rangle=0.013$ and the mass-load is given by $\langle\psi\rangle \rho_{p} / \rho_{g}=19.5$

tangential component of the relative velocity will occur. The criterion to determine the type of collision on basis of pre-collision information is as follows:

$$
J_{t}= \begin{cases}-\frac{2}{7}\left(1+\beta_{0}\right) m_{a b} \mathbf{v}_{a b, 0} \cdot \mathbf{t} & \text { if } \mu_{f} J_{n} \geq \frac{2}{7}\left(1+\beta_{0}\right) m_{a b} \mathbf{v}_{a b, 0} \cdot \mathbf{t} \\ -\mu_{f} J_{n} & \text { otherwise, }\end{cases}
$$

where the two equations respectively describe collisions of the sticking and sliding type. Given the definition of $\mathbf{J}$ in (17) and (18), the post-collision velocities can now be calculated from (9) and (10). In particle-wall collisions the mass of particle $b$ (i.e. the wall) is taken infinitely large, which makes all terms $1 / m_{b}$ equal to zero.

The particle collision characteristics play an important role in the overall system behavior as was shown by Hoomans et al. [24] and Goldschmidt et al. [19]. For this reason realistic collision properties of the particles are supplied to the model. The parameters used in the treatment of the discrete particles are summarized in Table 1. Here $\psi$ is the local solid volume fraction, which does not occur in the present model equations, but is computed for evaluation purposes (see Section 3.3). Collisions between particles are monitored as follows. For each particle, say A, a neighbor-list is kept. This includes all particles that are located within a certain radius of particle 'A'. Particles nearer the top of the list are closer to 'A', while particles that are further separated are stored lower on the list. After each collision among the entire particle swarm, the neighbor lists are updated when necessary.

\subsection{Subgrid-modeling}

In order to make large-scale turbulent flow simulations at high particle volume fractions feasible, the gas phase is described using large-eddy simulation. This is obtained by applying spatial filtering to the flow equations in order to reduce their dynamical complexity. The filter is defined by

$$
\bar{a}=\int G(x, \xi) a(\xi) d \xi
$$

where $\bar{a}$ denotes a filtered flow variable and $G$ the filter-kernel. For the compressible equations we use a density-weighted filter,

$$
\tilde{a}=\overline{\rho a} / \bar{\rho},
$$


the so-called Favre filter and originally proposed by Reynolds [51]. If the convolution filter is applied to the governing equations the result may be expressed in terms of the LES-template [17]: $\mathrm{NS}(\bar{U})=R(U, \bar{U})$ where the original and filtered variables are defined by $U=\left[\rho, u_{j}, p, T\right] ; \bar{U}=\left[\bar{\rho}, \widetilde{u}_{j}, \bar{p}, \widetilde{T}\right]$. The spatial filtering yields a 'closureresidual' $R(U, \bar{U})$ which contains, e.g., the filtered forcing term $\bar{f}_{i}$ and the divergence of the turbulent stress tensor

$$
\tau_{i j}={\overline{\rho u_{i} u_{j}}}-\overline{\rho u}_{i} \overline{\rho u}_{j} / \bar{\rho}=\bar{\rho}\left\{{\widetilde{u_{i} u_{j}}}-\tilde{u}_{i} \tilde{u}_{j}\right\}
$$

The only closure term modeled in this paper is this turbulent stress tensor. The subgrid terms that result from the filtering of the diffusive viscous fluxes are neglected. Likewise, subgrid contributions arising from filtering the momentum exchange between the discrete particles and the fluid as represented by the drag law are also neglected. This is a reasonable assumption for the present application, as we consider relatively coarse particles. The particles are slightly larger than the Kolmogorov length-scale and the Stokes response time is an order of magnitude larger than the Kolomogorov time. Therefore the motion of the particles is mainly influenced by the large-scale eddies in the flow [1, 28, 29].

Fevrier et al. [13] decomposed the instantaneous particle velocity field into a spatially correlated and a random (quasi-Brownian) component. They found that increase of particle inertia (increase of Stokes response time) leads to an increase of the quasi-Brownian component. Boivin et al. [4] and Fede and Simonin [9] showed that single point particle statistics are not sensitive to the fluid subgrid field in case the Stokes number is larger than 5. In the latter reference a particle diameter of 0.92 times the Kolmogorov length scale was used. It is unlikely that the conclusion would have been very different for slightly larger particles. Thus it appears reasonable to assume that in the present work, where the particle diameter equals 1.9 times the Kolmogorov length scale and the Stokes number based on Kolmogorov time is about 300, details of the fluid subgrid field do not influence particle statistics and the effects of particle collisions. Since in this paper, we focus on the effects of particle collisions, we model the fluid subgrid stress tensor like in unladen flow and ignore the subgrid term arising from the filtering of the particle force in the fluid equations. For much smaller Stokes numbers (around 3), the latter subgrid term was investigated by Okong'o and Bellan [47].

The simulations in this work have been performed using four different subgridmodels for the turbulent stress tensor $\tau_{i j}$, all applicable to unladen flow. As the simulation results were found to be quite insensitive of the adopted subgrid model, compared to the dynamic effects of the particles, we will only mention three of these models and provide some more detail for the model for which results will actually be shown. For details regarding the comparison of different subgrid models we refer to Vreman et al. [65, 68] and Geurts and Vreman [18].

The first three models, M1-3, are derived from the basic Smagorinsky model, which reads [54]:

$$
m_{i j}(\tilde{u})=-2 C_{S}^{2} \bar{\rho} \Delta^{2}|S(\tilde{u})| S_{i j}(\tilde{u}), \quad|S|=\left(\frac{1}{2} S_{i j} S_{i j}\right)^{\frac{1}{2}}, \quad \Delta=\left(\Delta_{1} \Delta_{2} \Delta_{3}\right)^{\frac{1}{3}},
$$

The symbol $\Delta_{i}$ denotes the filter width in the $i$-direction, assumed to be equal to the grid-spacing. The basic Smagorinsky model was extended in various ways. We 
included the following extensions as point of reference for assessing the dynamic contribution due to the particles:

- Filtered multi-scale model (M1): [57, 62], which is a generalization of the variational approach proposed by Hughes et al. [27]. By formulating the Smagorinsky model in terms of the filtered velocity fluctuations, the excessive dissipation associated with the Smagorinsky model in laminar and transitional flows is removed. This is in particular relevant close to the solid walls of the vertical channel. This model takes backscatter into account.

- Dynamic eddy-viscosity model (M2): which is based on the dynamic procedure $[14,33]$. This provides the possibility to calculate a 'Germano-optimal' eddyviscosity coefficient which adapts itself to the evolving flow. The standard dynamic model requires a number of explicit test-filtering operations.

- Taylor-approximated dynamic model (M3): which is based on a simplification of the dynamic procedure to avoid explicit filtering. The compressible anisotropic form was used [65] which extends the incompressible isotropic formulation [49]. The simplifications arising from Taylor expansions rely on the property: $\widehat{w}=\bar{w}+O\left(\Delta^{2}\right)[6,32]$. Chester et al. [5] and Verstappen [61] also formulated Taylor expansions of the dynamic model.

The standard dynamic eddy-viscosity and the Taylor approximated dynamic model may lead to negative eddy-viscosities and thus lead to ill-posed equations and unstable simulations, if the Reynolds number is sufficiently high. This problem can for example be solved by the ad-hoc procedures of ensemble averaging and/or simply clipping the negative values. A more elegant way to overcome this problem is to base the eddy-viscosity on a positive invariant of the gradient model $\beta_{i j}$, which is a positive definite tensor. This leads to model M4. Simulation results obtained with this model were found to be largely the same as obtained with any of the models M1-M3. Model M4 [63] employs the following positive invariant of the gradient model,

$$
B_{\beta}=\beta_{11} \beta_{22}-\beta_{12}^{2}+\beta_{11} \beta_{33}-\beta_{13}^{2}+\beta_{22} \beta_{33}-\beta_{23}^{2},
$$

and defines the eddy-viscosity

$$
v_{e}=c \sqrt{\frac{B_{\beta}}{\left(\partial_{j} \tilde{u}_{i}\right)\left(\partial_{j} \tilde{u}_{i}\right)}}
$$

where $c=0.07$. The dissipation of this eddy-viscosity and the exact subgrid dissipation were shown to vanish for precisely the same class of flows [63]. Model M4 was proposed independently of the work of Nicoud and Ducros [45], who constructed a similar eddy-viscosity. That eddy-viscosity is not based on the gradient model, but on the square of the velocity gradient matrix.

The use of several different subgrid-models allows to assess the sensitivity of the predictions. We found that the effects associated with the introduction of the discrete particles were much stronger than differences arising from a change in the subgrid model. For that reason, we will show explicit simulation results only for the eddyviscosity model that is based on the gradient model, i.e., M4. The other subgrid models were found to yield qualitatively and quantitatively similar results. 


\subsection{The numerical method}

The equations for the fluid phase are solved with a second-order finite volume method, based on central differencing on a collocated grid. For details we refer to Geurts and Kuerten [15] and Vreman et al. [67]. In the latter work, the second-order numerical method was found to be sufficiently accurate in LES, also with respect to the dissipation of kinetic energy.

The channel flow is solved on the domain $3 H \times 2 H \times 12 H$. The length of the domain in the spanwise $\left(x_{1}\right)$ direction is 1.5 times smaller than for most DNS/LES of single-phase channel flow in order to limit the amount of carried particles and their collisions to a manageable number. As a point of reference, we also performed a single-phase DNS for this computational domain. Specifically, we used an incompressible Fourier/Chebyshev method with $64 \times 128 \times 128$ modes. The mean and rms profiles (shown in the next subsection) were verified to be identical to those of standard DNS-databases for $\operatorname{Re}_{\tau}=180$ (e.g., [26, 42, 44, 60]).

The large-eddy simulations presented in this paper involve $32 \times 64 \times 64$ grid cells. The grid is only nonuniform in the normal direction and symmetric with respect to the plane $x_{2}=0$. The grid-points in the left-half of the channel are defined by

$$
x_{2, j} / H=-1+\frac{\sinh \left(a j / N_{2}\right)}{\sinh (a / 2)} \text { with } j=0, . ., N_{2} / 2 \text { and } a=6.5
$$

Verstappen and Veldman [60]. The first grid point of the wall is at $x_{2,1}=0.2 \mathrm{~mm}$, corresponding to $y^{+}=1.5$. The grid is sufficiently fine to have a well-resolved LES of channel flow at $R e_{\tau}=180$, according to common criteria (see [48]).

In this work the volume of the smallest computational fluid grid cell is still about 27 times larger than the volume of a particle. For the Euler-Lagrange method to be applicable, the fluid grid cell volume should preferably be an order of magnitude higher than the particle volume. With the present parameters this condition is hard to satisfy in DNS, however in LES it is easily satisfied. The mapping of properties from the Lagrangian particle positions to the nonuniform Eulerian computational grid and vice versa can be done in a straightforward manner through volume weighing techniques [7, 24]. For evaluation purposes the particle volume fraction $\psi$ is determined, by counting the particles within each cell of an auxiliary grid. In contrast to the Eulerian grid the auxiliary grid is uniform and contains $32 \times 25 \times 64$ cells. Thus, in each direction the mesh-spacing of the auxiliary grid is considerably larger than the particle diameter [24].

The discretization in time is explicit: forward Euler for the particles and the fluid viscous terms, and a four-stage Runge-Kutta scheme for the fluid convective and pressure terms, using coefficients $\frac{1}{4}, \frac{1}{3}, \frac{1}{2}$ and 1 . The values for the time-steps for the solids - and gas-phase need to be sufficiently small to accurately capture the different physical effects. However, working with too small time-steps may unnecessarily add to the computational cost without significantly increasing the overall accuracy. Hence, a proper balance between these constraints needs to be determined. For the fluid equations the time-step needs to be small enough to comply with numerical stability restrictions of the Runge-Kutta method, with the characteristic turbulent time-scales and with the acoustic waves arising at the low Mach number considered here. Regarding the time-step for the solids-phase, the Stokes response-time $\tau_{p}$ sets an important time-scale. In order to accurately determine the motion of the 
individual particles between collisions, the time-step needs to be taken well below $\tau_{p}$. Finally, since the solids- and gas-phase are treated with separate algorithms which represent different contributions to the overall cost, one has the freedom to adopt separate time-steps for the two coupled sub-systems, provided all aspects of the resolved flow-physics are accurately generated. Within this set of constraints, the time step is taken $10^{-4} \mathrm{~s}$ for the solids phase and $2 \cdot 10^{-5} \mathrm{~s}$ for the gas phase. Correspondingly, one Stokes response-time is divided into 4,000 time-steps of the solids-phase, which appears more than sufficient within the smoothed LES context. The time-step for the gas phase was taken a fraction of the numerical stability timestep, which was found to be more restrictive than other characteristic time-scales associated with the gas-phase. In total these values yield very small time-integration errors between particle collisions. The most costly part of the simulation method remains the discrete particle model, which requires about $80 \%$ of the computation time in case of four-way coupled simulations.

The simulations run until at least $t=5 \mathrm{~s}$, while statistics are accumulated between $t=3 \mathrm{~s}$ and $t=5 \mathrm{~s}$. The averaging time of $2 \mathrm{~s}$ corresponds to $20 H / u_{\tau}$ in terms of the wall shear-velocity $u_{\tau}$, twice as large as a typical averaging time in single-phase channel flow. With a Stokes response-time of $\tau_{p}=0.4 \mathrm{~s}$, the particles are evolved for $12.5 \tau_{p}$ and the accumulation of the statistics is over $5 \tau_{p}$. The particles considered in our simulation are highly inertial and in order to obtain quantitatively precise statistics, the averaging should preferably be over a more extended period, even up to tens of $\tau_{p}$. Averaging over shorter time-intervals will give rise to some statistical errors which may express themselves, e.g., as asymmetries in the average profiles of gas and solids properties such as velocity and turbulence intensities. Conversely, these asymmetries provide a first indication of the statistical averaging error. In the case studied in this paper we observed quite low levels of asymmetry already after averaging over only $5 \tau_{p}$ (this may be inferred from the profiles presented in the next section). Correspondingly, the main features of the average profiles and the influence of the solids-phase on these properties can be clearly discerned, despite the remaining statistical error. As the averaging process is known to converge quite slowly as function of the length of the averaging interval, it would be quite impractical to simulate for long enough for the statistical error to become virtually negligible. However, for the purpose of assessing and discerning the effects of two- and fourway coupled discrete particles, the adopted averaging interval appears well justified.

The partial derivatives in the subgrid model and those in the molecular viscous terms are treated in the same manner. The explicit filtering operations in the standard dynamic and multi-scale subgrid models are discretizations of the top-hat filter in each direction, using the trapezoidal integration rule on three points.

\section{Results}

In this section we will compare results obtained in 'clean' riser-flow with the particleladen case, using four different subgrid models. First we will consider the clean and four-way coupled cases and quantify the turbulence modulation of the gas phase arising from the presence of the particles (Section 3.1). In Section 3.2 we will isolate the effects of the particle collisions and compare the four-way coupled results 
with two-way coupled simulations. Finally we will show the occurrence of coherent particle structures in our simulations (Section 3.3).

\subsection{Turbulence modulation}

It appears that the particle-phase strongly alters the fluid mean flow. Figure 1 shows the fluid mean flow profile normalized with $U_{m}$ and $u_{\tau}$. Relative to the clean channel we observe that the particles give rise to a strongly reduced boundary layer thickness and a flatter velocity profile. It corresponds to a larger skin-friction coefficient and, consequently, a larger $R e_{\tau}$ based on the fluid velocity, which increases from 180 to 300. The effects of the embedded particles on the developing flow are also reflected by the profile in the logarithmic region. Compared to the clean case an approximately logarithmic velocity profile develops for $10^{-3}<x_{2}<10^{-2}$, i.e., corresponding to $20<y^{+}<200$, but at a much larger Von Kármán 'constant' [21]. In addition, the wall shear stress, proportional to the derivative of the mean gas flow at the wall, is increased. This derivative increases, because the gas velocity near the wall increases. Through the drag-force, the gas near the wall gains momentum from the particles which come from the center of the channel into the boundary layer (see also [64]). These particles loose their inertia slowly, because the Stokes response time is large. The gravity, being opposite to the flow direction also decelerates the particles, and this may explain the shoulder in the mean profile around $y=-0.85 H$ (Fig. 1a).

In single phase channel flows it is usual to normalize velocity statistics with $u_{\tau}$, which determines the fluid wall shear stress. In the present two-phase channel flow, the external pressure gradient $p_{g}$ balances the sum of wall shear stress of the fluid phase, the friction between wall and particles and the gravity force on the fluid and the particles. For a sufficiently high solid mass load the gravity on the particles dominates the other contributions and then normalization of velocity statistics with $u_{\tau}$ is less obvious. Therefore, Fig. 1 also shows the fluid mean flow normalized with $U_{m}$. In the following all velocity statistics are normalized with $U_{m}$, which is the same for all calculations.

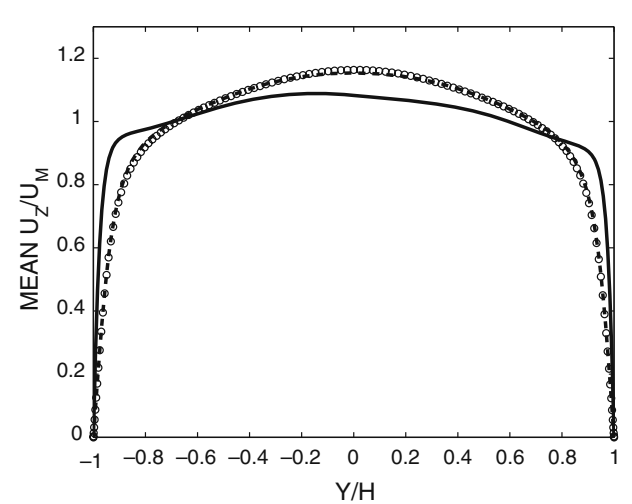

(a)

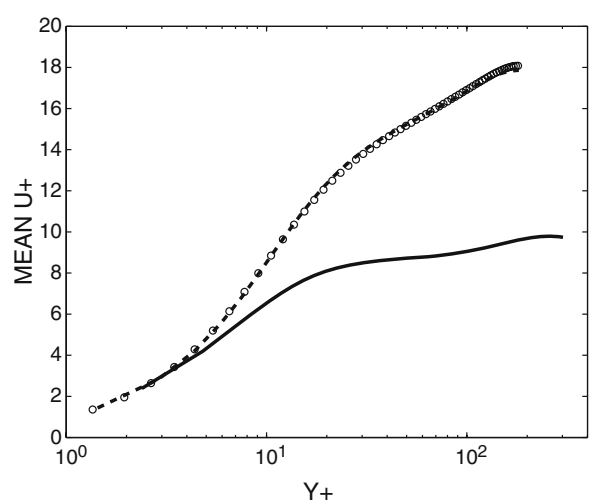

(b)

Fig. 1 Mean streamwise fluid velocity $\left\langle u_{z}\right\rangle$ : linear (a) and logarithmic (b) for a particle-laden flow, comparing clean flow (dashed) with four-way coupling (solid). Subgrid model M4 is used and DNS results of clean flow are indicated by circles 
Fig. 2 Turbulence intensities of the fluid phase; streamwise (top), normal (middle) and spanwise direction (bottom), comparing clean flow (dashed) with four-way coupling (solid). Subgrid model M4 is used and DNS results of clean flow are indicated by circles
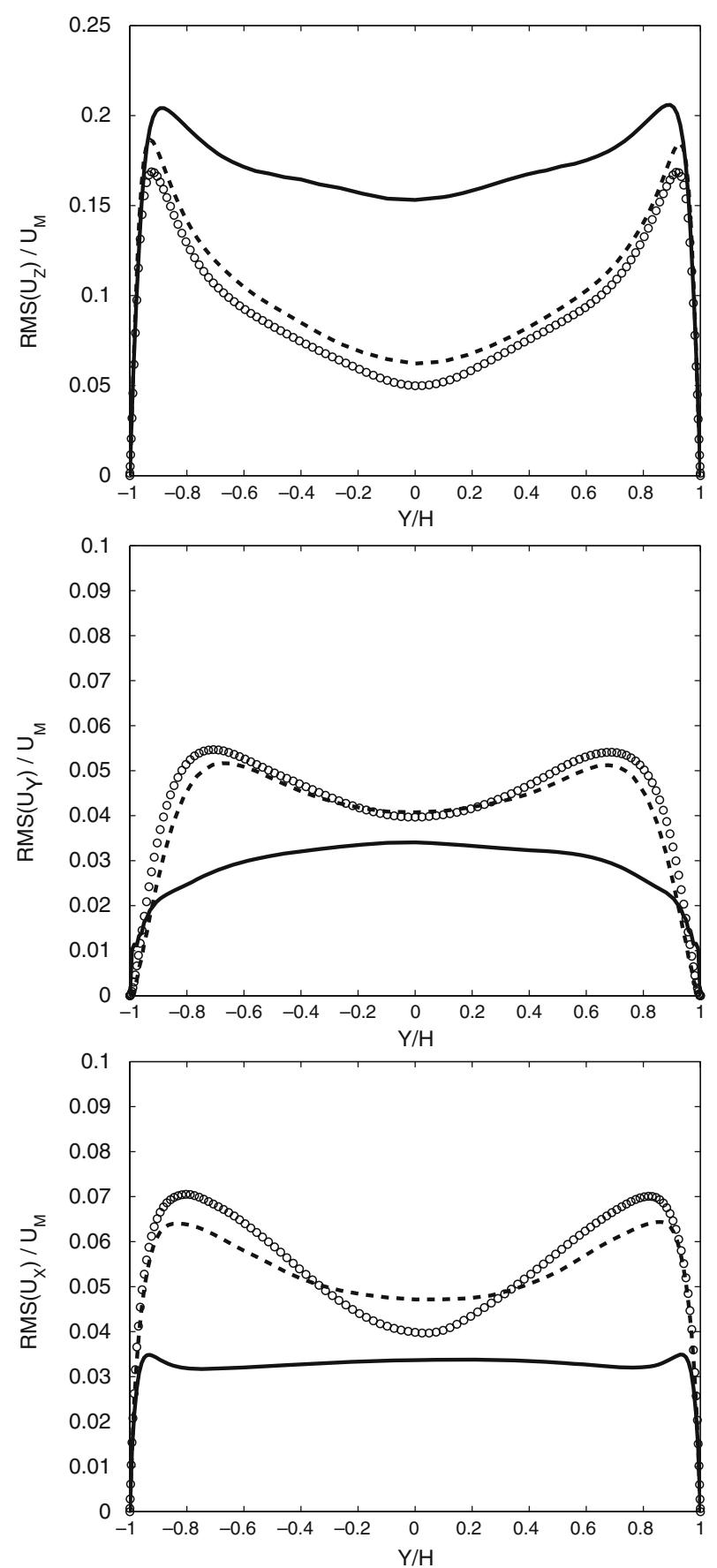

Figure 2 shows the turbulence intensities of the fluid phase. The turbulence modulation by coarse particles leads to an increased streamwise turbulence intensity and decreased transverse and spanwise intensities. This observation appears 
generally in line with existing experimental data. Although a direct comparison with physical experiments faces important difficulties in view of differences in flowconditions, volume fractions and particle properties, an interesting analogy with the simulation findings may be drawn. The experiments by Kulick et al. [30] adopt smaller particles at lower volume fraction. In this regime a decrease in all components of the turbulence intensity was noted. This appears to contrast the present simulation findings. However, in a study by Tsuji et al. [59] larger particles at higher volume fraction were studied including particles of $0.5 \mathrm{~mm}$ in diameter, with Stokes responsetime $\tau_{p} \approx 0.6 \mathrm{~s}$. This situation is close to our simulation setting in a number of respects. In such cases an increase in streamwise intensities in the core region of the channel was reported. For still coarser particles Tsuji et al. [59] found an even stronger increase of turbulence intensity across the entire diameter of the pipe. For a further and more detailed analysis of turbulence modulation we refer to Vreman [64].

The quality of the LES results may be established by comparison with DNSdata. The LES-results for the reference 'clean' channel flow at $R e_{\tau}=180$ are quite close to the DNS-results as far as the mean streamwise fluid velocity predictions are concerned (Fig. 1). The results for the mean streamwise fluid velocity $\left\langle u_{z}\right\rangle$ were found to display a limited variation with the adopted subgrid model.

A more sensitive assessment of the quality of LES predictions may be obtained by considering rms-fluctuation levels. Compared to the unfiltered DNS data, the streamwise intensity of the large-eddy simulation with M4 is slightly over-predicted when normalized with $U_{m}$. It is well-known that streamwise velocity fluctuations are somewhat over-predicted compared to DNS data, in case eddy-viscosity models, including proper near-wall damping, are employed ([29] and references therein). This was found in combination with a variety of numerical methods, e.g., high order finite volume methods but also in spectral discretizations. The normal and spanwise velocity fluctuations correspond quite closely to DNS predictions. In addition, some numerical under-resolution may contribute to the remaining overall error-level, although according to the commonly accepted criteria as expressed by Piomelli and Balaras [48], the grid is sufficiently fine to have a 'well-resolved' LES of channel flow. Such an under-resolution might induce a nonlinear accumulation of simulation errors arising from the interaction between discretization and subgrid modeling errors $[16,40,41]$; here these effects are sufficiently small for our purposes.

The turbulence intensities in the particle-laden cases are somewhat more sensitive to the subgrid model than in the clean channel case. This may be related to the fact that the boundary layer is thinner and the amplitude of the streamwise turbulence intensity is higher. In the particulate channel flow $\operatorname{Re}_{\tau}$ was observed to increase from 180 to a value around 300. Correspondingly, since the LES filterwidth was not changed, the dynamical effects of the sub-filter scales increases in the particulate flow and the specific closure for the turbulent stresses will have a stronger influence on the simulation results. This also implies that differences between the subgrid models become more pronounced and emphasizes the increased importance of accurate modeling of the turbulent stress tensor $\tau_{i j}$ in particle-laden flow. We observe some weak asymmetries in the results, which would probably only vanish if a much longer averaging time was adopted. Some additional improvement of the statistical convergence might be obtained by also averaging over both channel halves. 
However, in view of the large-scale coherent structures that arise in the particulate flow, such a procedure could yield approximate running-time averages of which the statistical error might be misinterpreted as too low. In fact, the degree of asymmetry between the time-averaged results in the two channel halves is a first independent indication of the remaining statistical error. Therefore, only the time-averaging was included. Note that the simulation time in terms of dimensionless time units is large, $50 H / u_{\tau}$. The slow convergence of the statistics is caused by the relatively large value of $\tau_{p}$, which measures the response-time of a particle to the surrounding flow.

In all cases the differences caused by the inclusion of particles are much larger than the observed asymmetries or the differences caused by the variation of the subgrid model. Thus the qualitative conclusions on the modulation of turbulence due to the inclusion of interacting particles are not contaminated by these effects.

\subsection{Effects of collisions}

In this subsection we quantify the effects of the collisions between particles. For this purpose we present a detailed comparison of the predictions of the four-way with the two-way coupled simulation.

In Fig. 3 we collected the mean streamwise fluid and solids velocity profiles. The comparison of two- and four-way coupling displays large qualitative differences to which we turn next.

Considering the mean flow, both the two- and four-way coupling cause a higher skin-friction coefficient and results in a near-wall profile quite similar to the fourway coupled case. The correspondence of the near-wall fluid velocity profiles in the two- and four-way coupled descriptions reflects the interaction of the particles with the solid channel walls which is identical in both cases. As observed above, the inelastic collisions with the walls create a low-velocity-layer directly adjacent, which effectively acts analogous to an increased wall-roughness and hence yields an increased skin-friction coefficient. The absence of inelastic particle-particle collisions

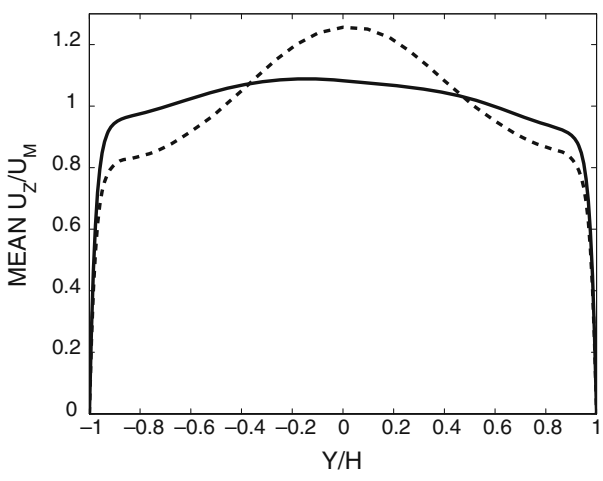

(a)

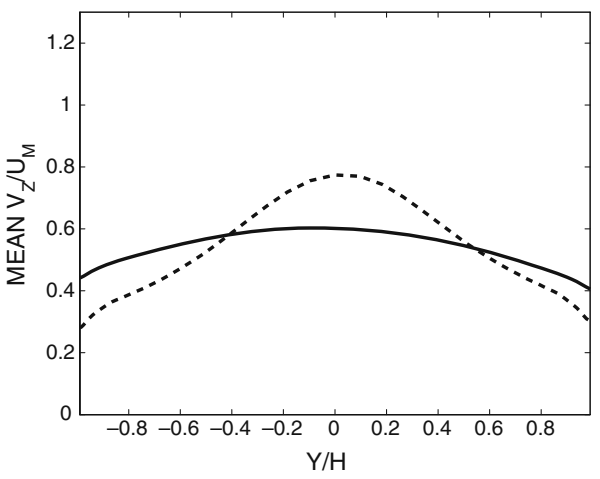

(b)

Fig. 3 Mean fluid velocity (a) and mean solids velocity (b), comparing two-way (dashed) with fourway coupling (solid) using subgrid model M4 
in the bulk of the flow is responsible for the absence of a flattening of the fluid velocity profile. The prediction of the bulk flow away from the boundary layers is quite different when comparing the two-way and the four-way approaches. The twoway description is seen to give rise to a somewhat localized 'center-jet' in which the fluid velocity is up to about $60 \%$ larger than the velocity at the edge of the boundary layer. In contrast, the four-way coupling gives rise to a slightly flatter velocity profile compared to the clean channel; the particle-particle collisions evidently allow to avoid the 'center-jet' as discussed above.

The consequences of collisions for the mean solids profile is shown in Fig. 3b. The strong center-jet in $\left\langle u_{z}\right\rangle$ observed in the two-way coupling model, also arises in $\left\langle v_{z}\right\rangle$. The shape of the four-way coupled solids velocity profile is much flatter and quite similar to experimental data [59]. We also observe that the near-wall solids velocity is positive and does not drop to zero (except when the distance between the measuring point and the channel wall is smaller than the particle radius). This is consistent with the observation that the mean fluid velocity profile in the boundary layer is enhanced by the forces of the particles on the fluid. It confirms experimental data with measurements below $y^{+}=40$ in a horizontal channel [52]: close to the wall particles move faster than the fluid, while in the outer layer the opposite occurs.

The striking differences between the resulting dynamics in the two-way and the four-way coupled descriptions of the solids-phase are directly related to the interparticle collisions. The reason is that these collisions diffuse kinetic energy of the solids in the normal direction. As a consequence the mean solids velocity profile flattens. Due to the coupling between phases through the drag force, this collisional diffusion also flattens the gas mean velocity profile.

Figure 4 shows the turbulence intensities of the two phases. Again there are large differences between two- and four-way coupling. In particular the transverse and spanwise intensities of the solids velocity are much lower if only two-way coupling is used. To achieve convergence of the statistics is more difficult for two-way coupling than for four-way coupling. This is indicated by the relatively large asymmetry of the profiles for the two-way coupled results. Also the normal spanwise fluid intensity in the case of two-way coupling shows some spurious oscillations near the boundary.

The particle volume fraction distribution is shown in Fig. 5. To obtain the nearwall details of $\langle\psi\rangle$ we did not use the instantaneous $\psi$ on the uniform auxiliary grid, but averaged the concentration of particles in vertical slices of the nonuniform Eulerian grid. A characteristic turbophoresis effect is visible in terms of an approximately $15 \%$ higher concentration near the solid walls. Turbophoresis has been observed in many experiments [73] and simulations. However the factor by which the particle concentration near the wall is increased, relative to the average bulk-value, depends strongly on the precise flow-regime that is considered. The relatively small turbophoresis effect observed in the present simulations may be attributed to the fact that the particles are coarse and the mass load is high. To verify this, we performed a four-way coupled simulation with particles with a much smaller diameter $\left(d_{p}=\right.$ $0.04 \mathrm{~mm})$ and, consequently, a lower particle concentration $\left(\psi=1.3 \cdot 10^{-5}\right)$. In that case strong turbophoresis was observed; the particle concentration near the walls increased with a factor of 30 relative to the mean bulk-concentration. When twoway coupling is used in combination with coarse, slowly responsive particles, no appreciable turbophoresis remains as is seen in Fig. 5. 

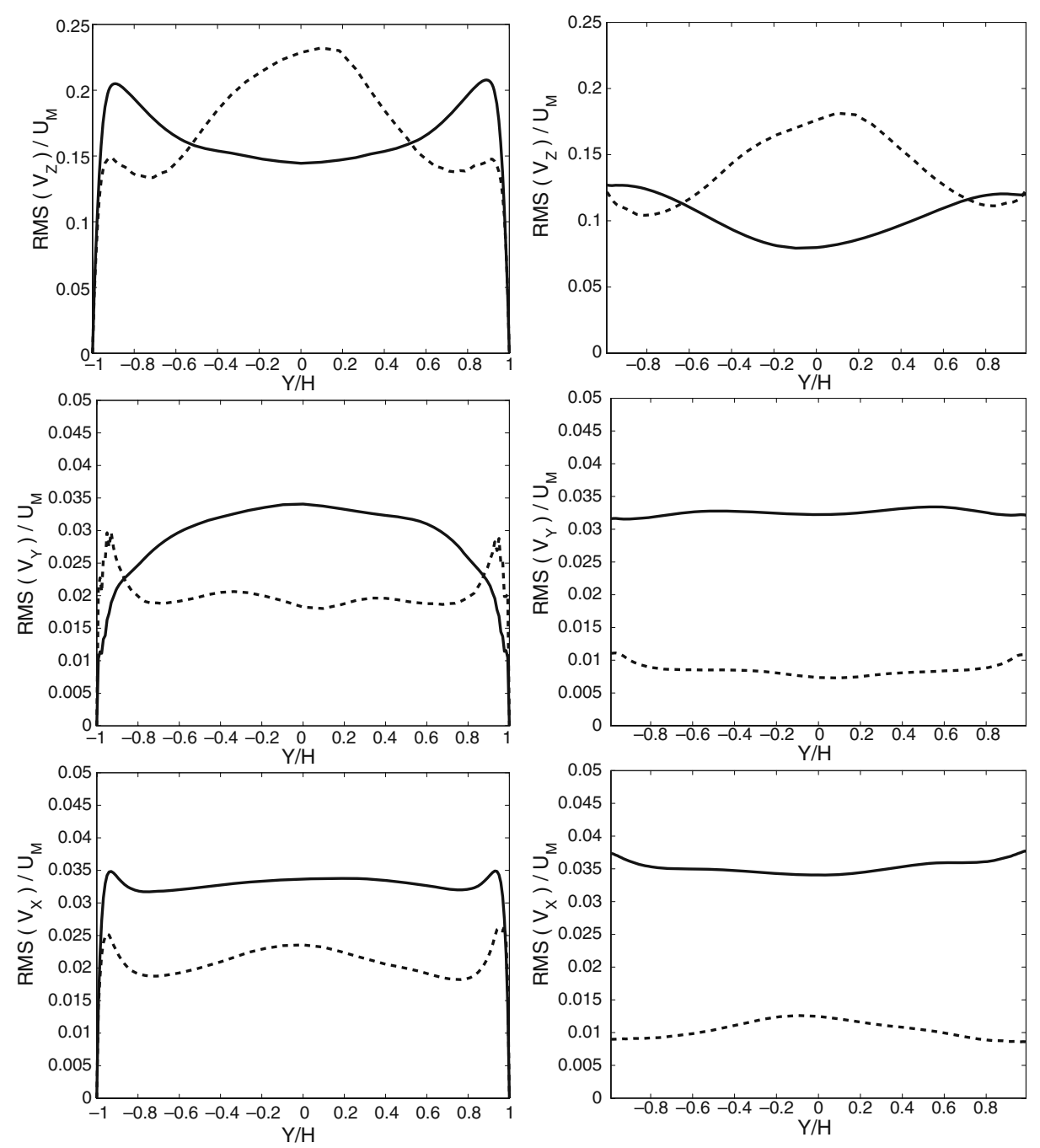

Fig. 4 Turbulence intensities for the fluid phase (left) and for the solids phase (right); streamwise (top), normal (middle) and spanwise direction (bottom), comparing two-way (dashed) with four-way coupling (solid) using subgrid model M4

\subsection{Coherent particle structures}

In this subsection we will consider the dynamic self-organization that arises due to the 'competition' between the structuring associated with the inelastic particle collisions and the bursting of particle-clusters due to the underlying tendency of the clean flow to develop strong turbulence, described by the dynamic eddy-viscosity model M2. The observed flow-structuring displays an interesting dynamic behavior which 
Fig. 5 Solid volume fraction $\langle\psi\rangle$ obtained with LES using subgrid model M4: two-way (dashed) compared with four-way coupling (solid). Notice that $\langle\psi\rangle$ is shifted downward by 0.005 for the two-way results for clarity

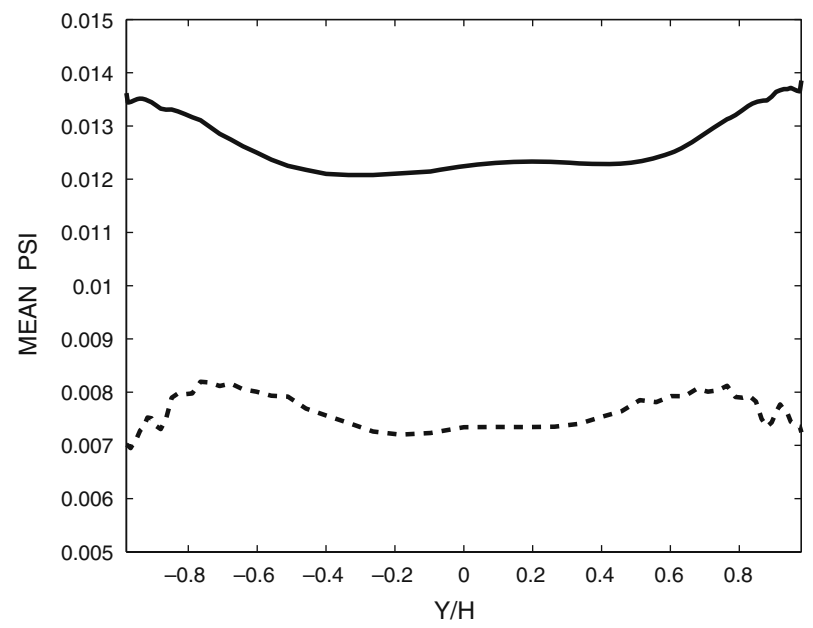

will be illustrated in terms of characteristic instantaneous snapshots of the particle concentration. These display qualitatively the sequence of formation and destruction of quite large coherent regions of considerably increased particle densities. This general flow-structure illustration complements the mean-flow and turbulence intensity results discussed in the previous subsections. We will also show that the four-way coupling model gives rise to large-scale coherent particle swarms which are much weaker when the two-way coupling model is used.

In order to characterize the flow-structuring we concentrate on visualizing the instantaneous particle volume fraction $\psi$ available at the uniform auxiliary grid. As point of reference, the basic riser flow was simulated using LES with the standard dynamic model and four-way coupling. The grid on which $\psi$ is evaluated contains $32 \times 25 \times 64$ cells. We show the particle volume fraction at different times in Fig. 6 . A contour value of $\psi=0.03$ is selected, while the solid volume fraction attains a maximum of approximately 0.1 and an average of 0.013 . From these snapshots one may infer the formation large-scale coherent structures of particle concentration.

The self-organization seen in Fig. 6 also arises when the other three subgrid models are used. As such, the dynamic self-organization of the particles is a robust phenomenon. At the particle volume fractions considered here, the use of the full four-way coupling is essential. This is illustrated in Fig. 7 in which we compare a structured particle field associated with four-way coupling, with a structure-less field arising in the two-way coupling model. These qualitative impressions further establish that four-way coupling can not be replaced by the computationally more appealing two-way coupling.

The four-way coupled simulations are seen to contain relatively dense regions of particles, transported by the mean flow and gradually changing with time. Without collisions these regions are considerably smaller and less dense (Fig. 7b), and therefore we conclude that the particle-particle interactions play a crucial role in the formation of coherent particle structures. 


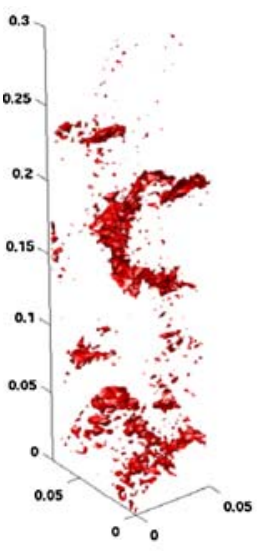

(a)

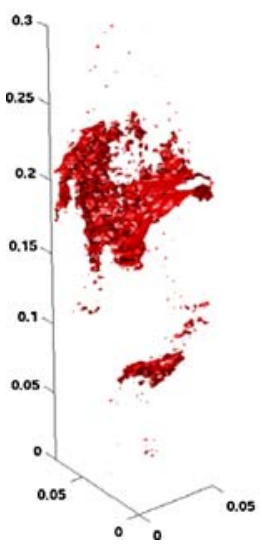

(e)

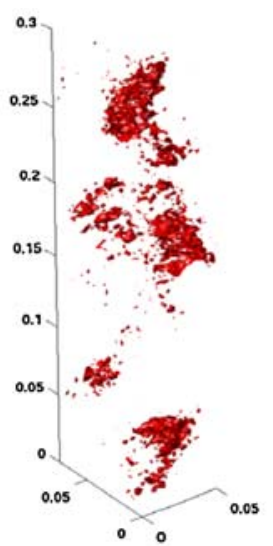

(b)

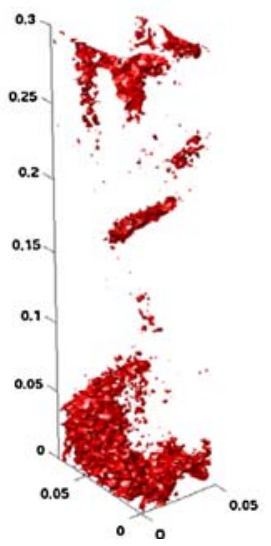

(f)

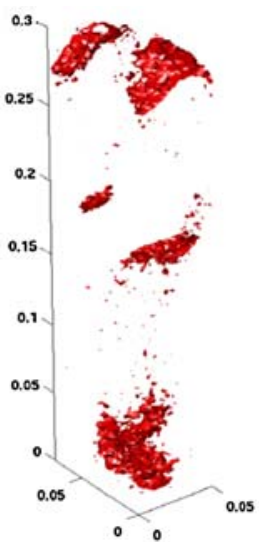

(c)

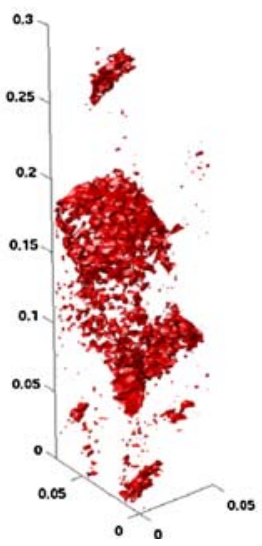

(g)

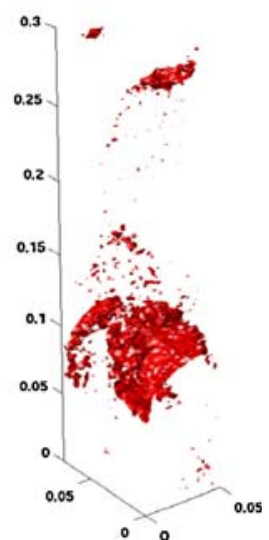

(d)

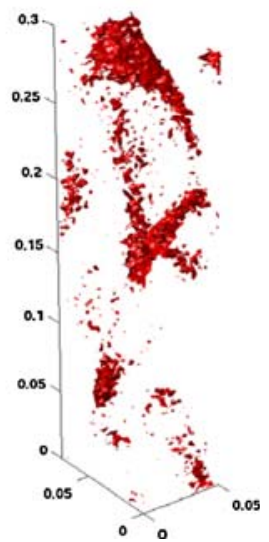

(h)

Fig. 6 Snapshots of the particle volume fraction showing iso-surfaces at $\psi=0.03$ for the dynamic eddy-viscosity model M2 with four-way coupling; $t=3.1 \mathrm{~s}$ (a) with steps of $0.05 \mathrm{~s}$ until $t=3.45 \mathrm{~s}$ (h)

Inelasticity of the collisions probably plays an important role in the clustering process: an inelastic collision decreases the velocity difference between the two particles involved, thus after the inelastic collision the probability that these two particles remain near to each other is higher than before the collision. The relation between inelastic collisions and the formation of clusters was investigated experimentally for sand-jets [36]. In addition, traveling waves of coherent particle structures were observed in the two-dimensional simulations by Liss et al. [35]. Although a dominant longitudinal wavelength is clearly visible in the present simulations as well, the structures are less organized than those visualized by Liss et al., an obvious effect of the three-dimensionality of the fluid turbulence in the present work. 
Fig. 7 Granular clustering in coherent particle-swarms is strongly associated with the four-way coupling description. Snapshot of the particle volume fraction at $t=4 \mathrm{~s}$ for the Taylor-approximated model M3 comparing the four-way coupling (a) with the two-way coupling (b). The iso-surfaces shown correspond to $\psi=0.03$

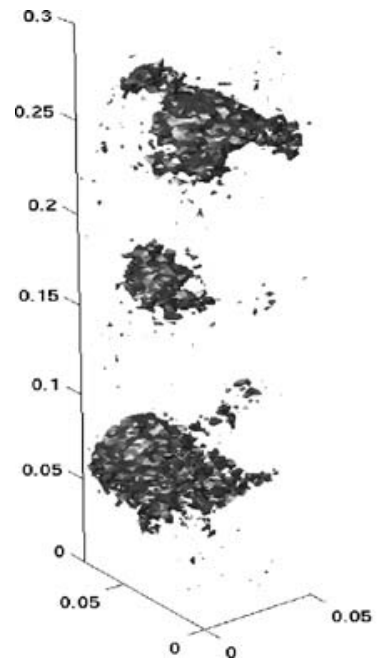

(a)

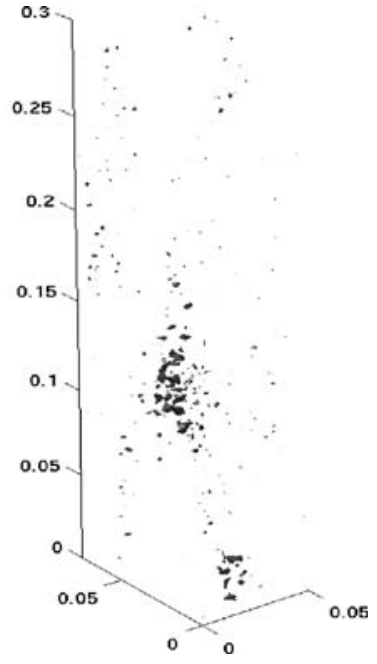

(b)

\section{Concluding Remarks}

In this paper we presented large-eddy simulation results of particle-laden turbulent flow in a vertical riser. This flow is relevant, e.g., to chemical processing and an understanding of the fundamental dynamics of this flow is essential in order to properly predict up-scaling of processes from a laboratory scale to settings which are of industrial importance. We showed that already at a modest particle volume fraction of about $1.5 \%$ the particle-particle interactions play an important role in the development of the flow. The computationally more accessible two-way coupling model proved to give rise to predictions, which for slowly responsive particles and the present particle volume fraction, lack a turbophoresis effect and show the occurrence of a fairly strong 'center-jet' which was not recorded in experimental studies. The present particle volume fraction is much larger than in previous studies of plane channel flow, and the effects of inter-particle collisions found in the present work are much larger than the collisional effects reported by Yamamoto et al. [69], who found a mild effect of collisions in their case.

The presence of a large number of interacting particles leads to a strong modulation of the turbulence in the channel. Relative to a clean channel the coupling between particles and fluid is mainly responsible for the reduction in the thickness of the boundary layer and the corresponding strong increase in the skin-friction. Moreover, the log-layer that is characteristic of wall-bounded flows was seen to be retained in the particle-laden case but with a much larger Von Kármán 'constant'. Turbulent intensities in the normal and spanwise directions were reduced whereas the streamwise turbulent intensity was found to be amplified by the presence of coarse particles. The conclusions regarding turbulence modulation in the plane channel flow are generally consistent with the experimental findings for coarse particles embedded in turbulent pipe flow.

In addition, the particle-particle interactions in the form of inelastic collisions are mainly responsible for retaining turbophoresis in case particles with high Stokes 
response-time are used, and a flattening of the mean particle velocity distribution. These interactions also gave rise to the occurrence of dynamic self-organization of the embedded particles in coherent swarms.

Acknowledgements This work was sponsored by the Stichting Nationale Computerfaciliteiten (National Computing Facilities Foundation, NCF) for the use of supercomputer facilities, with financial support from the Nederlandse Organisatie voor Wetenschappelijk Onderzoek (Netherlands Organization for Scientific Research, NWO). A. W. Vreman is grateful to the Turbulence Program of the Dutch foundation for fundamental research of matter (FOM), and to the Institute for Mechanics, Process-engineering and Control Twente (IMPACT), for financial support. Fruitful discussions with J. M. Link are acknowledged, assisting with the discrete particle model and we are indebted to N. D. Sandham for providing the initial perturbations for turbulent channel flow.

Open Access This article is distributed under the terms of the Creative Commons Attribution Noncommercial License which permits any noncommercial use, distribution, and reproduction in any medium, provided the original author(s) and source are credited.

\section{References}

1. Armenio, V., Piomelli, U., Fiorotto, V.: Effect of the subgrid scales on particle motion. Phys. Fluids 11, 3030 (1999)

2. Armenio, V., Fiorotto, V.: The importance of the forces acting on particles in turbulent flows. Phys. Fluids 13, 2437 (2001)

3. Bagchi, P., Balachandar, S.: Effect of turbulence on the drag and lift of a particle. Phys. Fluids 15, 3496-3513 (2003)

4. Boivin, M., Simonin, O., Squires, K.D.: On the prediction of gas-solid flows with two-way coupling using large-eddy simulation. Phys. Fluids 12, 2080-2090 (2000)

5. Chester, S., Charlette, F., Meneveau, C.: Dynamic model for LES without test filtering: quantifying the accuracy of Taylor series approximations. Theor. Comput. Fluid Dyn. 15, 165-181 (2001)

6. Clark, R.A., Ferziger, J.H., Reynolds, W.C.: Evaluation of subgrid-scale models using an accurately simulated turbulent flow. J. Fluid Mech. 91, 1 (1979)

7. Delnoij, E., Kuipers, J.A.M., van Swaaij, W.P.M.: A three-dimensional CFD model for gas-liquid bubble columns. Chem. Eng. Sci. 54, 2217-2226 (1999)

8. Elghobashi, S., Truesdell, G.C.: On the two-way interaction between homogeneous turbulence and dispersed solid particles. I: Turbulence modification. Phys. Fluids A 5, 1790-1801 (1993)

9. Fede, P., Simonin, O.: Numerical study of the subgrid fluid turbulence effects on the statistics of heavy colliding particles. Phys. Fluids 18, 045103 (2006)

10. Feng, Z.-G., Michaelides, E.E.: Proteus: a direct forcing method in the simulations of particulate flows. J. Comp. Phys. 202(1), 20-51 (January 2005)

11. Ferrante, A., Elghobashi, S.: On the physical mechanisms of two-way coupling in particle-laden isotropic turbulence. Phys. Fluids 15, 315-329 (2003)

12. Ferry, J., Balachandar, S.: A fast Eulerian method for disperse two-phase flow. Int. J. Multiph. Flow 27, 1199-1226 (2001)

13. Fevrier, P., Simonin, O., Squires K.: Partioning of particle velocities in gas-solid turbulent flows into a continuous field and a spatially uncorrelated random distribution: theoretical formalism and numerical study. J. Fluid Mech. 533, 1-46 (2005)

14. Germano, M., Piomelli, U., Moin, P., Cabot, W.H.: A dynamic subgrid-scale model. Phys. Fluids A 3, 1760-1765 (1991)

15. Geurts, B.J., Kuerten, J.G.M.: Numerical aspects of a block-structured flow solver. J. Eng. Math. 27, 293 (1993)

16. Geurts, B.J., Fröhlich, J.: A framework for predicting accuracy limitations in large-eddy simulation. Phys Fluids 14, L41-L44 (2002)

17. Geurts, B.J.: Elements of Direct and Large-Eddy Simulation. Edwards Publishing Inc. ISBN: 1-930217-07-2 (2003)

18. Geurts, B.J., Vreman, A.W.: Dynamic self-organization in particle-laden turbulent channel flow. Int. J. Heat Fluid Flow 27, 945-954 (2006) 
19. Goldschmidt, M.J.V., Beetstra, R., Kuipers, J.A.M.: Comparison of the kinetic theory of granular flow with 3D hard-sphere discrete particle simulations. Chem. Eng. Sci. 57, 2059-2075 (2002)

20. Gore, R.A., Crowe, C.T.: Effect of particle size on modulating turbulence intensity. Int. J. Multiph. Flow 15, 279-285 (1989)

21. Hinze, J.O.: Turbulence: an Introduction to its Mechanism and Theory. McGraw-Hill, New York (1975)

22. Van der Hoef, M.A., Beetstra, R., Kuipers, J.A.M.: Lattice Boltzmann simulations of low Reynolds number flow past mono- and bi-disperse arrays of spheres: results for the permeability and drag force. J. Fluid Mech. 528, 233-254 (2005)

23. Van der Hoef, M.A., van Sint Annaland, M., Deen, N.G., Kuipers, J.A.M.: Numerical simulation of gas-solid fluidized beds: a multiscale modeling strategy. Ann. Rev. Fluid Mech. 40, 47-70 (2008)

24. Hoomans, B.P.B., Kuipers, J.A.M., Briels, W.J., van Swaaij, W.P.M.: Discrete particle simulation of bubble and slug formation in a two-dimensional gas-fluidized bed: a hard-sphere approach. Chem. Eng. Sci. 51, 99-118 (1996)

25. Hoomans, B.P.B.: Granular dynamics of gas-solid two-phase flows. PhD-Thesis, University of Twente (1999)

26. Howard, R.J.A., Sandham, N.D.: Simulation and modeling of a skewed turbulent channel flow. Flow Turbul. Combust. 65, 83-109 (2000)

27. Hughes, T.J.R., Mazzei, L., Jansen, K.E.: Large eddy simulation and the variational multi-scale method. Comput. Vis. Sci. 3, 47 (2000)

28. Kuerten, J.G.M.: Subgrid modeling in particle-laden channel flow. Phys. Fluids 18, 025108 (2006)

29. Kuerten, J.G.M., Vreman, A.W.: Can turbophoresis be predicted by large-eddy simulation? Phys. Fluids 17, 011701 (2005)

30. Kulick, J.D., Fessler, J.R., Eaton, J.K.: Particle response and turbulence modification in fully developed channel flow. J. Fluid Mech. 277, 109-134 (1994)

31. Lakehal, D., Smith, B.L., Milelli, M.: Large-eddy simulation of bubbly turbulent shear flows. J. Turbul. 3, 25 (2002)

32. Leonard, A.: Energy cascade in large-eddy simulation of turbulent fluid flows. Adv. Geophys. 18, 237 (1974)

33. Lilly, D.K.: A proposed modification of the Germano subgrid-scale closure method. Phys. Fluids A 4, 633 (1992)

34. Li, Y., McLaughlin, J.B., Kontomaris, K., Portela, L.: Numerical simulation of particle-laden turbulent channel flow. Phys. Fluids 13, 2957-2967 (2001)

35. Liss, E.D., Conway, S.L., Glasser, B.J.: Density waves in gravity-driven granular flow through a channel. Phys. Fluids 14, 3309-3326 (2002)

36. Lohse, D., Bergmann, R.P.H.M., Mikkelsen, R., Zeilstra, C., Meer, R.M., van der Versluis, M., Weele, J.P., van der Hoef, M.A., van der Kuipers, J.A.M.: Impact on soft sand: void collapse and jet formation. Phys. Rev. Lett. 93 198003-1-198003-4 (2004)

37. Marchioli, C., Giusti, A., Salvetti, M.V., Soldati, A.: Direct numerical simulation of particle wall transfer and deposition in upward turbulent pipe flow. Int. J. Multiph. Flow 29, 1017-1038 (2003)

38. Mathiesen, V., Solberg, T., Hjertager, B.H.: An experimental and computational study of multiphase flow behavior in a circulating fluidized bed. Int. J. Multiph. Flow 26, 387-419 (2000)

39. Maxey, M.R., Riley, J.: Equation of motion for a small rigid sphere in a turbulent fluid flow. Phys. Fluids 26, 883 (1983)

40. Meyers, J., Geurts, B.J., Baelmans, M.: Database-analysis of errors in large-eddy simulations. Phys. Fluids 15, 2740 (2003)

41. Meyers, J., Geurts, B.J., Baelmans, M.: Optimality of the dynamic procedure for large-eddy simulation. Phys. Fluids 17, 045108 (2005)

42. Moin, P., Kim, J.: Numerical investigation of turbulent channel flow. J. Fluid Mech. 118, 341 (1982)

43. Moran, J.C., Glicksman, L.R.: Mean and fluctuating gas phase velocities inside a circulating fluidized bed. Chem. Eng. Sci. 58, 1867-1878 (2003)

44. Moser, R.D., Kim, J., Mansour, N.N.: Direct numerical simulation of turbulent channel flow up to Ret $=590$. Phys. Fluids 11, 943-945 (1999)

45. Nicoud, F., Ducros, F.: Subgrid-scale stress modeling based on the square of the velocity gradient tensor. Flow Turbul. Combust. 62, 183-200 (1999)

46. Nieuwland, J.J.: Hydrodynamic modeling of gas-solid two-phase flows. PhD-Thesis, University of Twente (1995) 
47. Okong'o, N.A., Bellan, J.: Consistent large-eddy simulation of a temporal mixing layer laden with evaporating drops. Part 1. Direct numerical simulation, formulation and a priori analysis. J. Fluid Mech. 499, 1-47 (2004)

48. Piomelli, U., Balaras, E.: Wall-layer models for large-eddy simulations. Ann. Rev. Fluid Mech. 34, 349 (2002)

49. Pope, S.B.: Turbulent Flows. Cambridge University Press (2000)

50. Powers, J.M.: Two-phase viscous modeling of compaction of granular materials. Phys. Fluids 16, 2975-2990 (2004)

51. Reynolds, O.: On the dynamical theory of incompressible viscous fluids and the determination of the criterion. Phil. Trans. 186, 123 (1895)

52. Righetti, M., Romano, G.P.: Particle-fluid interactions in a plane near-wall turbulent flow. J. Fluid Mech. 505, 93-121 (2004)

53. Sagaut, P.: Large Eddy Simulation for Incompressible Flows. Springer Verlag (2001)

54. Smagorinsky, J.: General circulation experiments with the primitive equations. Mon. Weather Rev. 91, 99 (1963)

55. Squires, K.D., Eaton, J.K.: Particle response and turbulence modification in isotropic turbulence. Phys. Fluids A 7, 1191-1203 (1990)

56. Squires, K.D., Simonin, O.: Recent advances and perspective of DNS and LES for dispersed twophase flow. In: Proceedings of the 10th Workshop on Two-Phase Flow Predictions, Merseburg, pp. 152-163 (2002)

57. Stolz, S., Schlatter, P., Meyer, D., Kleiser, L.: High-pass filtered eddy-viscosity models for LES. In: Friedrich, R., Geurts, B.J., Metais, O. (eds.) Direct and Large-Eddy Simulation V: Muenchen. pp. 81-88. Kluwer, Dordrecht (2004)

58. Tryggvason, G., Bunner, B., Esmaeeli, A., Juric, D., Al-Rawahi, N., Tauber, W., Han, J., Nas, S., Jan Y.-J.: A front-tracking method for the computations of multiphase flow. J. Comput. Phys. 169, 708-759 (2001)

59. Tsuji, Y., Morikawa, Y., Shiomi, H.: LDV measurements of an air-solid two-phase flow in a vertical pipe. J. Fluid Mech. 139, 417-434 (1984)

60. Verstappen, R.W.C.P., Veldman, A.E.P.: Symmetry-preserving discretization of turbulent flow. J. Comput. Phys. 187, 343-368 (2003)

61. Verstappen, R.W.C.P.: A synthesis of similarity and eddy-viscosity models. In: Friedrich, R., Geurts, B.J. Metais, O. (eds.) Direct and Large-Eddy Simulation V: Muenchen. pp. 89-96. Kluwer, Dordrecht (2004)

62. Vreman, A.W.: The filtering analog of the variational multi-scale method in large-eddy simulation. Phys. Fluids 15, L61-64 (2003)

63. Vreman, A.W.: An eddy-viscosity model for turbulent shear-flow: algebraic theory and applications. Phys. Fluids 16, 3670-3681 (2004a)

64. Vreman, A.W.: Turbulence characteristics of particle-laden pipe flow. J. Fluid Mech. 584, 235279 (2007)

65. Vreman, A.W., Geurts, B.J., Deen, N.G., Kuipers, J.A.M.: Large-eddy simulation of a particleladen turbulent channel flow. In: Friedrich, R., Geurts, B.J., Metais, O. (eds.) Direct and LargeEddy Simulation V, pp. 271-278. Kluwer, Dordrecht (2004)

66. Vreman, B.: Direct and large-eddy simulation of the turbulent compressible mixing layer. PhDThesis, University of Twente (1995)

67. Vreman, B., Geurts, B., Kuerten, H.: Comparison of numerical schemes in large-eddy simulation of the temporal mixing layer. Int. J. Numer. Methods Fluids 22, 297 (1996)

68. Vreman, B., Geurts, B.J., Kuerten, J.G.M.: Large-eddy simulation of the turbulent mixing layer. J. Fluid Mech. 339, 357-390 (1997)

69. Yamamoto, Y., Potthoff, M., Tanaka, T. Kajishima, T., Tsuji, Y.: Large-eddy simulation of turbulent gas-particle flow in a vertical channel: effect of considering inter-particle collisions. J. Fluid Mech. 442, 303-334 (2001)

70. Wang, Q., Squires, K.D.: Large eddy simulation of particle deposition in a vertical turbulent channel flow. Int. J. Multiph. Flow 22, 667 (1996)

71. Wen, Y.C., Yu, Y.H.: Mechanics of fluidization. Chem. Eng. Prog. Symp. Ser. 62, 100-111 (1966)

72. Whitaker, S.: The Forchheimer equation: a theoretical development. Transp. Porous Media 25, 27-61 (1996)

73. Young, J., Leeming, A.: A theory of particle deposition in turbulent pipe flow. J. Fluid Mech. 340, 129-159 (1997)

74. Zhang, D.Z., Prosperetti, A.: Momentum and energy equations for disperse two-phase flows and their closure for dilute suspensions. Int. J. Multiph. Flow 23, 425-453 (1997) 